\title{
EL CRITERIO INSTITUYENTE EN LAS CATALOGACIONES LITERARIAS EN CENTROAMÉRICA
}

\author{
Carlos Manuel Villalobos
}

\begin{abstract}
RESUMEN
Este artículo investiga cuáles han sido los mecanismos instituyentes en la elaboración de las antologías literarias de Centroamérica. Se parte de los primeros compendios de carácter regional y nacional publicados a finales del siglo XIX y se rastrea la trayectoria a lo largo del siglo XX e inicios del XXI. Los criterios de inclusión y exclusión de obras y autores trascienden los códigos estéticos y coinciden con el devenir de los imaginarios de nacionales y políticos del área.

Palabras clave: mecanismos instituyentes, antologías literarias, Centroamérica.
\end{abstract}

\begin{abstract}
This article investigates which have been the institutional mechainsms in the elaboration of the literary anthologies of Central America. As part of the first compendiums of regional character and national publication at the end of the nineteenth century continues the trajectory along the twentieth century a the beginning of the twenty-first. The criteria for the inclusion and exclusion of orks and authors transcends aesthetic codes and coincides with the coming of the imaginaries of the nationalists and politicians of the area.
\end{abstract}

Key words: instituional mechanisms, literary anthologies, Central America.

M.L. Carlos Manuel Villalobos. Profesor, de la Escuela de Filología, Lingüística y Literatura, Universidad de Costa Rica. San Pedro, San José, Costa Rica.

Recepción: 26-4-06

Aceptación: 25-5-06 
Toda antología es, por su naturaleza, el establecimiento de un canon y al mismo tiempo el desplazamiento de otros posibles. Quien hace la selección debe contar con la argucia del buen catador. Debe tener el buen gusto de un jardinero cuando selecciona las mejores flores para el arreglo. No es por una fosilización cursi que se proponen estas metáforas. Es porque en efecto, semánticamente, antología implica seleccionar flores (del griego , av日os, flor, y $\lambda \dot{\varepsilon} \gamma \varepsilon ı \nu$, escoger).

Una indagación de los mecanismos antológicos seguidos en Centroamérica permite visualizar mejor cuáles son las estrategias canónicas que han prevalecido en el área. Al mismo tiempo, esta revisión posibilita reflexionar sobre las justificaciones nacionalistas que activan los proyectos fundacionales de las letras patrias y los que posteriormente intentan enmendar o actualizar los ya establecidos.

Según la bibliografía que consigna Jorge Eduardo Arellano en las fuentes para el estudio de la literatura centroamericana (2003), el número de antologías publicadas en Centroamérica sobrepasa las doscientas ${ }^{1}$, pero con carácter exclusivamente centroamericano el número apenas sobrepasa las veinte.

De acuerdo con Arellano, las primeras tres antologías de carácter centroamericano se publicaron todas en el año de 1888, pero al precisar los datos de edición se pudo comprobar que en realidad el año de la primera publicación es 1882. Se trata de El parnaso centroamericano del guatemalteco José García Salas. Mientras tanto Galería poética centroamericana, de Ramón Uriarte, también publicada en Guatemala, sí corresponde al año 1888. Aparte de estas recopilaciones guatemaltecas, un trabajo similar se publica en Nicaragua en 1888. Se trata de una antología que reúne fragmentos de autores centroamericanos. El autor es Pedro Ortiz y el trabajo se titula Frutos de lo nuestro ${ }^{2}$. Sin embargo, esta publicación no es tan ambiciosa como las de Salas y Uriarte, y resulta una excepción en el marco de la relevancia por las antologías de carácter nacional que se publicaban en diferentes países ${ }^{3}$ durante este mismo contexto.

La siguiente etapa de la historia antológico-literaria centroamericana es liderada por el hondureño Rafael Heliodoro Valle, historiador y prolífero divulgador del pensamiento latinoamericano. En 1914, Heliodoro Valle elabora la primera antología poética centroamericana del siglo $\mathrm{XX}^{4}$. Este trabajo previo le abre las puertas de las Ediciones Ercilla en Chile, quienes divulgan antologías contemporáneas latinoamericanas y concuerdan con Valle una nueva antología centroamericana para publicar en Sudamérica. La propuesta final del hondureño se sale del criterio contemporáneo que tenía la serie, pero el editor de todas maneras decide su publicación como una forma de divulgar la totalidad de la poesía centroamericana. Esta pauta divulgativa es también la estrategia que mueve al hondureño a seleccionar un amplio grupo de 96 poetas que se consignan sin distingo de nacionalidad, excepto Rafael Landívar que encabeza la lista y entre paréntesis se apunta que es guatemalteco.

Es por esto mismo que el propio Valle niega que este compendio sea antología, es decir selección, "sino un ensayo que pretende fijar límites para valoraciones futuras". "La intención primordial -agrega más adelante- no ha sido la de llamarlos a certamen, sino la de presentar un panorama" (1941:15). El concepto de "índice”, bajo un criterio más o menos cronológico, (hay algunas incongruencias) resulta una estrategia bastante hábil para eximirse de los reclamos por exclusiones que suelen preceder a la publicación de toda antología, máxime cuando, en este caso, es el primer compendio de poemas centroamericanos que se publican fuera de la región. 
Para defender el valor literario del amplio número de incluidos, Valle convoca al poeta Arturo Mejía Nieto, quien se encarga de escribir el prefacio, y, de paso, contradecir al propio Valle cuando anota: "Aquí se incluye lo que ha parecido mejor al antologista (sic)" (1941:9). Para la defensa de esta producción centroamericana, Mejía Nieto argumenta que al igual que la flora, la poesía de esta zona ha sido exuberante. El ejemplo obligado es Rubén Darío, cuya nacionalidad queda establecida claramente como centroamericana, pues "no ha sido nuestro único gran poeta de la América Central. Precisamente de la época del maestro a nuestros días, buena agua ha corrido debajo de los puentes y en ella se ha reflejado un nuevo paisaje como motivo primordial del inspiración" (1941:11). Lo autóctono constituye el nuevo fundamento lírico que orienta la producción del área: “interesa por igual velar nuestra raíz telúrica, y descubrir nuestra voz, perdida entre tantos ruidos" (Idem). Esta acotación funciona además como una advertencia para explicar porqué algunos de los poemas rompen con los esquemas de la retórica modernista y se atreven a la libertad de alusiones locales más libres. Por eso muchos de los poemas, según Mejía Niego, huelen a guayabas, pinos y a flores de campo entre otros elementos de la campiña. (1941:12).

La vocación antológica centroamericanista encuentra entre 1948 y 1950, en el Salvador, un último foco de interés en la primera mitad del siglo XX. Saúl Flores publica un compendio de lecturas centroamericanas y Hugo Lindo edita en dos tomos la Antología del cuento moderno centroamericano (1949-1950). Si tomamos en cuenta las dos antología de Heliodoro Valle, la de Flores y este tomo doble de Hugo Lindo, en total en la primera mitad del siglo XX, solo se publicaron cuatro antologías con criterio centroamericano, lo que evidencia que el interés centroamericanista era bastante reducido. No así en la segunda mitad donde el porcentaje de publicaciones fue mucho mayor. En cuanto a los géneros, la mayoría de las antologías habían privilegiado la poesía o fragmentos modélicos de distingos géneros. La antología de Lindo, es la primera dedicada exclusivamente al relato corto.

En 1960, en el marco del Primer Festival del Libro Centroamericano, y como parte de la Organización Continental de Festivales del Libro, con sede en Bogotá, Colombia, se publican dos nuevas antologías centroamericanas: una dedicada a la poesía y la otra al cuento. Como director del festival funge el propio Miguel Ángel Asturias, pero las antologías se confeccionan con base en los aportes de aliados estratégicos en cada uno de los países. Se excluyen adrede algunos nombres significativos como Darío, Juan Ramón Molina, Arévalo Martínez y Salarrué, dado que figuraban en volúmenes especiales anteriores.

La idea de reunir, para un festival latinoamericano, a todos los países centroamericanos, permite inferir la interpretación del área como región literaria. Este imaginario, desde afuera, será constante en otras antologías centroamericanas que se divulgan otros países latinoamericanos, España y Estados Unidos 5 .

Una de las últimas antologías centroamericanas publicadas en el área es la actualización de una propuesta que data del año 1995 del guatemalteco Francisco Albizúrez Palma, esta vez publicada en Costa Rica en el año 2003. En el prólogo Albizúrez, propone una caracterización de "Centroamérica" a partir de las coincidencias y diversidades, tanto desde el punto de vista histórico como literario. Procede luego a comparar dos verosímiles estéticos: el modernismo y la contemporaneidad. Finalmente indaga las variantes y direcciones de la poesía centroamericana y concluye con una nota sobre el papel relevante de la poesía femenina: "Me parece de justicia elemental dejar constancia de la rica presencia de la mujer en la poesía contemporánea de Centroamérica" (2003:34). 
En cuanto a la narrativa, la última antología de dimensión ístmica es la compilación Pequeñas resistencias 2. Antología del cuento centroamericano contemporáneo de Enrique Jaramillo Levi, publicada en Madrid en el año 2003. Jaramillo recopila 60 cuentos (10 autores por cada país $)^{6}$. El libro contiene además un estudio introductorio elaborado por el antólogo en el que intenta inscribir la narrativa de la región en el ámbito latinoamericano y procurar dar cuenta de las particularidades de la cuentística centroamericana. El mismo título "resistencias", ofrece una pista que muestra una parte del carácter de la literatura del istmo. En este rasgo, Jaramillo Levi encuentra una explicación al marcado interés que suscita en este momento la literatura centroamericana. Los medios de comunicación social y las grandes editoriales empiezan a interesarse por lo que sucede en Centroamérica precisamente a partir de la situación conflictiva que ha marcado la historia reciente del área. No obstante, a lo largo del siglo XX, no ha sido posible una divulgación efectiva de esta narrativa. Según Jaramillo Levi

\footnotetext{
...la pobreza y subdesarrollo de la región, la falta de editoriales y la pésima distribución de libros, la escasez de críticos literarios profesionales, las dificultades de los propios autores para viajar y darse a conocer fuera de sus países y, en muchos casos, una generalizada mediocridad de las obras escritas, la cual empaña la visibilidad de un creciente número de textos realmente destacados (2003:16-7).
}

Sin duda, criterio de lo regional literario sostiene esta significativa cantidad de antologías centroamericanas, las que intentan no solo sistematizar una canonización interna, sino mostrar internacionalmente los modelos más destacados de un área que, a través de estas antologías, intenta darse a conocer como integrada.

\section{Los inventarios nacionales en el siglo $\mathrm{XX}$}

Entre los primeros antólogos que elaboraron recopilaciones nacionales destacan Félix Medina, quien en 1873, bajo el título de Lira Nicaragüense, elaboró el primer compendio literario del área7; Román Mayorga Rivas, editor de Guirnalda salvadoreña (El Salvador 18841886) y Máximo Fernández quien reunió los poemas de La lira costarricense (1890-1891).

En Honduras, quien elabora una primera recopilación antológica es el poeta Rómulo Durón. En 1896, editó Honduras Literaria, dedicado a los Escritores en prosa y tres años después, en 1899, divulga una segunda parte alusiva a la lírica: Escritores en verso. La publicación Honduras Literaria, además, es el primer proyecto no solo de divulgación, sino también fundacional de los estudios literarios hondureños.

En cuanto a Guatemala, lo único que se editó con identidad nacional (entendiendo por ello, la patria chica) a finales del siglo XIX fueron dos compendios dedicados a biografías de literatos elaborados por Antonio Batres Jáuregui ${ }^{8}$.

En el caso de Panamá, en cuanto logra su independencia en 1903, procura de una vez participar en la configuración de las antología nacionales. Una de las primeras referencias que intenta dar cuenta de la literatura de esta zona es el Parnaso Istmeño, publicado en este país en 1904, bajo la dirección de Donaldo Velasco.

La intención tácita, y a veces explícita, de estas primeras antologías fue consolidar una imagen del espacio nacional frente a lo extranjero, y al mismo tiempo instituir valores propios. Estos proyectos iniciaron la canonizan y la descatalogación de autores y obras en los respectivos países. Fueron además, la primer tentativa de un trabajo crítico-historiográfico en función de lo nacional. Trascienden los linderos estéticos y asumen una dimensión política implícita 
en la proyección de la imagen nacional. Esta explicación coincide con los señalamientos de la crítica académica costarricense, a propósito de la antología de Máximo Fernández (Quesada, 1986; Rojas y Ovares, 1995; y Chen, 1996). Estos estudiosos concuerdan en que La lira costarricense constituyó una primera formulación de lo nacional literario. Esta afirmación es válida para los demás proyectos, pues coinciden en un tiempo finisecular y en un espacio de escisiones territoriales que intentan afirmarse frente a los demás países, excepto Guatemala que orientó sus esfuerzos a unificar culturalmente el área a través de la literatura.

La intención política de los inventarios literarios nacionales de estos países centroamericanos, también se pueden hallar en la propuesta guatemalteca. Pero en este caso, la estrategia es una apuesta de poder que apela a los resabios de la historia centroamericana, al proponerse como depositaria e instituyente del espíritu literario de la patria grande.

A partir de la segunda década del siglo XX es posible establecer una nueva etapa significativa en la elaboración de antologías nacionales. Es el auge en los parnasos hispanoamericanos que se divulgan en España mediante antologías editadas en Madrid y Barcelona. La primera antología de esta naturaleza en publicarse en Europa es la de Nicaragua (1912), le sigue la de El Salvador (1916) y Costa Rica con una en 1920 y otra en $1921^{9}$.

En el caso de Guatemala y Panamá, aunque originalmente publicaron los parnasos en los respectivos países, poco después consiguieron también un espacio editorial en Europa. El único país del área que se queda por fuera en este contexto de antologías parnasianas es Honduras.

El Parnaso panameño, elaborado por Octavio Méndez Pereira, se publica en 1916 y, junto con el Parnaso ístmico, ya citado, de Donaldo Velasco, constituyen dos proyectos fundacionales historiográficos que intentan sistematizar la literatura de este país. El trabajo de Méndez Pereira da pie a uno más amplio, (Antología panameña) publicado en 1926 en el que se incluye tanto lírica como prosa. Sin embargo, no es este proyecto el que se publica en Europa. De esta tarea se encargará Demetrio Korsi, quien publica Antología de Panamá Parnaso y Prosa, en Barcelona en 1926.

En relación con el parnaso guatemalteco, Humberto Porta Mencos es el encargado publicarlo en Guatemala en $1928^{10}$. En Barcelona se edita en 1930 de modo que, casi al filo de la eclosión parnasiana, Guatemala logra ingresar a las páginas de las antologías líricas latinoamericanos que se difundían en Europa.

Como parte del proyecto de difusión nacionalista, Porta Mencos, el compilador, encuentra oportuno conectar la poesía guatemalteca con el prestigio de Rafael Landívar. Sin embargo, en la edición de 1977, se incluye de primero el himno guatemalteco, escrito por el cubano José Joaquín Palma, con lo que se articula un ideario mucho más doméstico que el de 1928.

Estos prontuarios líricos cumplen una doble función. Por un lado, satisfacen el imaginario enciclopédico de los europeos; y por otro, aseguran el carácter civilizado que opera como requisito para la adquisición del estatus nacional en las representaciones culturales del momento.

Los procesos constitutivos de los estados nacionales centroamericanos, encuentran en estos inventarios un requisito básico del verosímil letrado. A través de las antologías logran mostrar los altos valores de la cultura. En 1938, Rogelio Sotela, publica una antología de literatura costarricense a la que agrega un anexo de biografías. No solamente se procura conservar la obra, sino que además se intenta que el nombre del autor perdure como modelo nacional. Sotela lo dice claramente en el prólogo: 


\begin{abstract}
He aquí los nombres ilustres de la patria naciente; representan ellos la inicial de la cultura nuestra y vienen aquí, no a que les demos prestigio en estas páginas de antología, sino a brillar noblemente para que el libro se prestigie con sus nombres preclaros (Sotela 1938: 3).
\end{abstract}

El crítico nicaragüense, Leonel Delgado, reflexiona sobre los mecanismos ideológicos que sustentan la producción antológica en Nicaragua. Coincide que se mantiene íntegra la idea nacional (patriótica o de identidad) como cimiento de la labor canonizadora. Pasa revista a un conjunto de antologías nicaragüenses recientes como La Poesía nicaragüense, de Ernesto Cardenal (1973); Soles de eternos días, de Anastasio Lovo y Erwin Silva (1999); Poesía política nicaragüense, de Francisco de Asís Fernández; Antología general de la poesía nicaragüense, de Jorge Eduardo Arellano (1984), e Hija del día: artes poéticas nicaragüenses, de Julio Valle-Castillo (1994). (2001:55).

Este corpus le permite a Delgado determinar que las canonizaciones en Nicaragua privilegian el género de la poesía por tener un significado más nacionalista. Aduce que esos criterios se unen al poco desarrollo que ha tenido la teoría, la historia y la crítica literaria.

Estos aspectos "oscurecen gran parte de las búsquedas y orientaciones, así como sus significados para la historia cultural” (2001:60). Más adelante, Delgado retoma este tema y aduce que

\footnotetext{
hablar de un debate literario en Nicaragua equivale casi siempre a ingresar a los subterráneos chismorreos y las batallas sordas. Se queja del poder cultural de la poesía bajo la unción de la tribu de Rubén, lo que ha propiciado una idea del escritor como "cantor divino colocado a la cabeza de las sociedades", no obstante que importantes creadores nicaragüenses más bien deberían figurar en la lista de los "ángeles exterminadores. (2002: 155).
}

En relación con el trabajo de Daysi Zamora, La mujer en la poesía nicaragüense, Delgado afirma que "tiene varios argumentos imprescindibles para la historia y la crítica literaria nicaragüenses, ante todo la idea del ingreso de las mujeres como sujetos en el ámbito literario y político, con un concepto de liberación más profundo e integral" (2001:62).

Una constatación muy sugerente en relación con la primacía canónica patriarcal es que, precisamente, las antologías que se apartan del esquema identificatorio nacional son las que integran con mayor cuidado las producciones femeninas.

Una explicación que resulta bastante lógica es que el interés de la antología ginésica es dar cuenta de la posibilidad femenina frente al poder histórico de la voz masculina y masculinizada. En cambio, el objetivo de las antologías nacionales es mucho más patrimonial, es decir, de consolidación hegemónica. Las antologías de mujeres saltan entonces como discursos contrahegemónicos y, en consecuencia, como reorientadoras del canon.

La exclusión que ha construido el canon hegemónico centroamericano no solamente implica al sujeto femenino, sino a otros sujetos sociales como por ejemplo a los indígenas, quienes permanecieron al margen de la comunidad letrada.

No obstante esta propuesta, Oviedo solo antóloga relatos publicados por las generaciones de escritores de 1970 y 1980. De este modo, lo que se proponía inicialmente como una propuesta de ruptura, termina en una sinopsis descriptiva y con acotaciones axiológicas. La antología no implica ninguna novedad en relación con las que la anteceden. Comparte, de este modo, los mismos principios que orientan la tradición historiográfica occidental que se ha seguido, en general, en Centroamérica. 
El costarricense Carlos Francisco Monge, quien también es antólogo, considera que en Costa Rica con excepción de Carlos Rafael Duverrán, “desde 1970 las antologías reproducían ciertas tachas: escasa información histórico-literaria, individualización de los hechos literarios, anecdotismo y en algunos casos un gusto por la polémica sin contendores" (2003:37). Sin embargo, muchos de los prólogos que anteceden los trabajos antológicos, incluyendo el propio trabajo de Monge, son meras aproximaciones historiográficas. De este modo, el reto que se propone superar quien elabora la escogencia de los autores, es fundamentalmente de carácter filial, es decir cómo explicar la pertenencia de un autor a un determinado movimiento, una generación o a una serie temática.

El inventario artístico tiende a demostrar las vinculaciones con lo nacional o regional. El antólogo participa, de este modo, en la construcción de los imaginarios de pertenencia (Sandoval 2003:9) que inciden en la formación del Estado-Nación. Mostrar el arte y demostrar con ello el espíritu cultural de la nación era más que una necesidad en el imaginario liberal y la ideología de la civilización ilustrada. Esta es al menos una de las intenciones básicas que parece motivar los trabajos antológicos elaborados en Centroamérica. La instituyente literaria, de este modo, consagra a unos y expulsa a otros: esa, finalmente, es la misión de quién escoge, al antojo, las palabras de su identidad.

\section{Notas}

1. Desde 1882 hasta la fecha, con alcance exclusivamente centroamericano se han publicado alrededor de 23 antologías, distribuidas de la siguiente manera: tres a finales del siglo XIX, dieciocho durante el XX y dos en lo que va del siglo XXI. El género privilegiado de estas antologías es la poesía, con un total de 12 recopilaciones poéticas, mientras que la narrativa ocupa un total de 8 proyectos, el teatro tiene uno, y hay al menos dos que incorporan escritos varios, donde se incluye el ensayo. Si lo vemos en términos porcentuales la poesía ocupa más de $50 \%$ de interés entre los antólogos centroamericanistas.

2. La obra se publicó en Managua en la Tipogafía de "El País". Constaba de 251 páginas. En el año de 1970 se reprodujo en la Revista Conservadora del Pensamiento Centroamericano. N $^{\circ} 170$.

3. Excepto Panamá, que aún no tenía estatuto nacional y, desde luego, Guatemala que le dio prioridad a las antologías centroamericanas y obvió las de carácter nacional.

4. Se trata de Poetas modernos de Centroamérica, publicado en Tegucigalpa.

5. Estas son algunas otras antologías centroamericanas publicadas fuera del área: Narradores centroamericanos (Ecuador, 1973) de Cristóbal Garcés Larrea; Poesía contemporánea de Centroamérica (España, 1983) de Roberto Armijo y Rigoberto Paredes; Volcán. Poems from Central America; (Estados Unidos, 1983) de Alejandro Munguía y Barbara Paschke; Contemporary short stories from Cental América (Estados Unidos, 1994) de Enrique Jarmillo Levy y Leland H. Chambers; y Cuentos centroamericanos (Chile, 2001) de Poli Délano.

6. Originalmente el proyecto era mucho más ambicioso y pretendía seleccionar un promedio de 25 autores por país, pero por razones editoriales tuvo que prescindir de varios nombres.

7. Esta primera antología se propone como una colección de los mejores ensayos de los poetas nicaragüenses. Medina incluye 6 poetas y consigna 18 poemas (Chinandega, Imprenta "Del Progreso", 1873. 43 p.). 
8. Se trata de los libros: Literatos guatemaltecos. (Landívar e Irrisari) Guatemala: Tipografía Nacional, 1889 y Biografías de literatos guatemaltecos. Guatemala: Academia Guatemalteca Correspondiente a la Real Academia Española: 1889.

9. Los datos bibliográficos de estas publicaciones fueron consignados por Eduardo Arellano en la exhaustiva información bibliográfica que consigna al final del Diccionario de Autores Centroamericanos, (2003). Los referencias son las siguientes: Alberto Ortiz. Parnaso nicaragüense. Antología completa de sus mejores poetas. Barcelona, Maucci, 1912. 253 p. (30 poemas y 137 poetas); Salvador L. Erazo: Parnaso salvadoreño. Antología esmeradamente seleccionada de los mejores poetas de la República de El Salvador. Barcelona, Maucci, 1917. 303 p. (34 poetas y 164 poemas); Eduardo de Oray. Los mejores poetas de Costa Rica. Madrid, Compañía Ibero-Americana de Publicaciones, 1920. 304 p.; Rafael Bolívar Coronado. Parnaso costarricense. Selección esmerada de los mejores poetas de Costa Rica. Barcelona, Maucci, 1921. 207 p. (15 poetas y 89 poemas).

10. Se trata del Parnaso Guatemalteco (1750-1928) editado por Humberto Porta Mencos (Guatemala, Tipografía Nacional, 1928. 560 p.). Una segunda edición, corregida y aumentada se publicó en Barcelona dos años después (Editorial Maucci, 1930). La última edición, que es la que se consultó para esta investigación se hizo en Guatemala en 1977 (Editorial José de Pineda Ibarra).

\section{Bibliografía}

Arellano, Jorge Eduardo. 2003. Literatura Centroamericana. Diccionario de autores centroamericanos. Managua: Fundación Vida.

Delgado Aburto, Leonel. 2002. Márgenes recorridos. Apuntes sobre procesos culturales y literatura nicaragüense del siglo XX. Managua, Instituto de Historia de Nicaragua y Centroamérica.

Durón, Rómulo E. 1958. Honduras Literaria. Tegucigalpa: Publicaciones del Ministerio de Educación Pública.

García Salas, José. 1882. El Parnaso Centroamericano. Guatemala: Imprenta de Pedro Arenales.

Jaramillo Levi, Enrique. 2003. Pequeñas resistencias 2. Antología del cuento centroamericano contemporáneo. Madrid : Editorial Páginas de Espuma.

Monge, Carlos Francisco. 1992. Antología de la poesía de Costa Rica. San José: Editorial de la Universidad de Costa Rica.

Ovares, Flora y otros. 1993. La Casa paterna. Estructura y nación en Costa Rica. San José: Editorial Universidad de Costa Rica.

Porta Mencos, Humberto. 1928. El Parnaso Guatemalteco. Guatemala: Tipografía Nacional. (Segunda edición corregida y aumentada. Barcelona: Editorial Maucci, 1930; Tercera edición. Guatemala: Ed. José de Pineda Ibarra, 1977). 
Quesada Soto, Álvaro. 1986. La formación de la narrativa nacional costarricense (18901910) Enfoque histórico social. San José: Editorial UCR.

Salas García, José. 1882. El Parnaso Centroamericano. Guatemala, Imprenta de Pedro Arenales. (y la reimpresión del Ministerio de Educación Pública, 1962, 2 vol.).

Sandoval García, Carlos. 2002. Otros amenazantes. Los nicaragüenses y la formación de identidades nacionales en Costa Rica. San José: Editorial UCR. 